\title{
Übertragbarkeit des spezifischen Wissens der Case Study
}

Die Übungsaufgaben beinhalten für die Studierenden Transferanforderungen, bei welchen sie über den alleinigen Fokus auf das konkrete Unternehmen Nibbles und den Müsli- und Cerealienmarkt hinausgehen sollen.

Nichtsdestotrotz empfiehlt sich, eine Bearbeitung der Case Study mit einer Diskussion der Übertragbarkeit des spezifischen Wissens dieser auf andere Unternehmensfälle und Kontexte abzuschließen. Dadurch werden die Bearbeitenden nochmals explizit dazu angeregt, das in der Case Study angeeignete Wissen auf andere Situationen und insbesondere ihren beruflichen Alltag zu transferieren. Die vorliegende Case Study gilt als exemplarische Vorgehensweise für jede Art der Produkteinführung oder Neuproduktentwicklung und soll dazu beitragen, theoretisches Wissen an einem praktischen Beispiel anwendbar zu machen.

Grundsätzlich sind der Prozess und die vorangehenden Marktanalysen einer Produkteinführung ebenso wie die Gestaltung des strategischen und operativen Marketings übertragbar und bieten damit eine fundierte analytische Grundlage für die Herangehensweise in Unternehmen.

Die spezifischen Anforderungen des Müsli- und Cerealienriegelmarktes bilden spezifisches Wissen und zeigen exemplarisch die Besonderheiten verschiedener Märkte, von deren Zielgruppen, den Wünschen der Konsumenten und der Produktvielfalt. 